\title{
Performance of the BEM solution in 3D acoustic wave scattering
}

\author{
A.J.B. Tadeu*, L. Godinho, P. Santos \\ Department of Civil Engineering, Faculty of Science and Technology, University of Coimbra - Polo II, Pinhal de Marrocos, 3030-290 Coimbra, Portugal
}

Received 12 January 2000; accepted 23 February 2001

\begin{abstract}
A fixed cylindrical circular cavity and a cylindrical circular column of fluid of infinite length submerged in a homogeneous fluid medium, and subjected to a pressure point source, for which closed form solutions are known, are used to assess the performance of constant, linear and quadratic boundary elements in the analysis of acoustic scattering.

This aim is accomplished by evaluating the error committed by the boundary element method (BEM) for a wide range of frequencies and wave numbers. First, the position of dominant BEM errors in the frequency versus spatial wave number domains are identified and related to the natural modes of vibration of the cylindrical circular inclusion. Then, the errors that occur by using constant, linear and quadratic elements are compared when the inclusion is modelled with the same number of nodes (i.e. maintaining computational cost). Finally, the importance of the position of the nodal points inside discontinuous boundary elements is analysed. (C) 2001 Elsevier Science Ltd. All rights reserved.
\end{abstract}

Keywords: Wave propagation; Acoustic scattering; Boundary element method; Two- and-a-half-dimensional problem; Boundary element errors

\section{Introduction}

Over the years, many researchers have found their attention drawn to the behaviour of waves propagating in a semifinite medium with discontinuities. Some of the earliest analytical studies on wave diffraction and scattering dealt with the problems of wave motion and reverberations in alluvial basins of regular shape [1], and of wave scattering induced by cavities [2,3]. More recently, semi-analytical methods have been used to analyse wave diffraction caused by geological irregularities of arbitrary shape within globally homogeneous media [4]. The application of purely numerical methods (i.e. finite elements or finite differences) has generally been restricted to situations where the response is required only within localised irregular domains, such as soil-structure interaction problems [5]. Discrete methods have also occasionally been used to model large alluvial basins, under plane-strain conditions [6].

The $2 \mathrm{D}$ acoustic scattering field caused by deformations of an oceanic waveguide's surfaces and by objects embedded between two half-spaces of different densities has been computed using a boundary integral equation method [7-9]. More recently, a boundary integral formula-

\footnotetext{
* Corresponding author. Tel.: +351-239-797-201; fax: +351-239-797190.

E-mail address: tadeu@dec.uc.pt (A.J.B. Tadeu).
}

tion to analyse acoustic barriers over an impedance plane as infinitely thin structures has been presented [10].

Hybrid methods involving a combination of finite elements to model the interior domain containing the inhomogeneities and semi-analytical representations for the exterior domain have also been employed [11], and a transitional matrix solution for the spectral scattering response of a partially buried 3D elastic obstacle in a plane stratified fluid media has been formulated [12].

Most of the above-mentioned numerical methods have only been applied in situations where the solution is required within 2D domains. Computationally demanding numerical schemes are required to evaluate the full scattering wave field generated by sources placed in the presence of 3D propagation media.

With a two-dimensional (2D) medium, the solution is much simpler, even if the dynamic source remains threedimensional (3D) (e.g. a point load). Such a situation is frequently referred to as a two-and-a-half-dimensional problem (or 2-1/2D for short), and solutions can be obtained for this by means of a 2 spatial Fourier transform in the direction in which the geometry does not vary. To do this, a sequence of $2 \mathrm{D}$ problems with different spatial wave numbers $k_{z}$ must be solved, after which the $3 \mathrm{D}$ field is synthesised using the inverse Fourier transform.

Although this solution is known in closed form for inclusions with simple geometry, such as a circular cylinder, for 
which the wave equation is separable, the solution is more difficult to obtain if the inclusion has an irregular cross-section. The boundary element method (BEM) may be the best tool to analyse wave propagation in unbounded media, as it satisfies the far-field radiation conditions automatically and permits a compact description of the medium in terms of boundary elements placed at the material's discontinuities [13-16].

The accuracy of the BEM solution depends on the number of boundary elements used to discretize the material discontinuities and on the nodes inside each element $[17,18]$. The BEM solution improves as the order of the element increases and its size decreases. However, the improvement in accuracy and efficiency that can be obtained by using a higher order entails a greater cost of CPU time. Thus, although the response improves with the number of nodes per element, this is not necessarily useful, as these more accurate models cost considerably more in terms of computational effort.

The present work assesses the benefit of using constant, linear and quadratic elements to analyse the 3D acoustic scattering. It does this by calculating the pressure in the vicinity of a circular cylindrical inclusion buried inside an acoustic medium, for which the solution is known in closed form. Simulations for both a fixed cylindrical cavity and a cylindrical column of fluid are conducted.

First, the equations required to solve the BEM problem are presented, and its analytical solution is given. Then, the BEM errors committed in the scattering analysis of an inclusion are identified in the frequency domain and related to the natural vibration modes. Different numbers of constant, linear and quadratic elements are used in these analyses, according to the differing ratio of the incident wave wavelength to the length of the boundary elements. To keep the computational cost essentially constant, comparisons are made between results computed with a similar number of nodal points. Finally, the performance of discontinuous linear and quadratic elements is analysed when the positions of the nodal points inside the boundary elements are moved around in the vicinity of those used in the Gauss-Legendre numerical integration.

\section{Problem statement}

Consider a cylindrical irregular inclusion of infinite extent, submerged in a spatially uniform fluid medium (Fig. 1), subjected to a harmonic point pressure source at position $\left(x_{0}, 0,0\right)$, oscillating with a frequency $\omega$

$p_{\text {inc }}=\frac{A \exp \left[i(\omega / \alpha)\left(\alpha t-\sqrt{\left(x-x_{0}\right)^{2}+y^{2}+z^{2}}\right)\right]}{\sqrt{\left(x-x_{0}\right)^{2}+y^{2}+z^{2}}}$,

where the subscript inc denotes the incident field, $A$ the wave amplitude, $\alpha$ the pressure wave velocity of the medium, and $\mathrm{i}=\sqrt{-1}$.

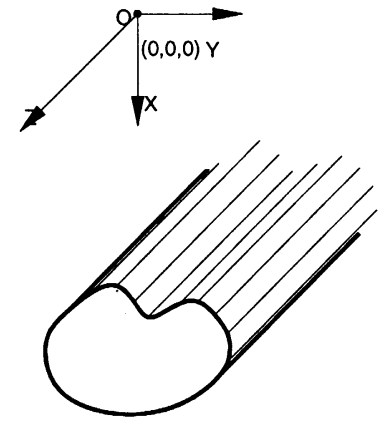

Fig. 1. Geometry of the problem.

Defining the effective wave numbers

$k_{\alpha}=\sqrt{\frac{\omega^{2}}{\alpha^{2}}-k_{z}^{2}}, \quad \operatorname{Im} k_{\alpha}<0$

by means of the axial wave number $k_{z}$, and Fourier-transforming Eq. (1) in the $z$ direction, we obtain

$\hat{p}_{\text {inc }}\left(\omega, x, y, k_{z}\right)=\frac{-\mathrm{i} A}{2} H_{0}^{(2)}\left(k_{\alpha} \sqrt{\left(x-x_{0}\right)^{2}+y^{2}}\right)$,

where $H_{n}^{(2)}(\ldots)$ are second Hankel functions of order $n$.

If an infinite set of periodically placed sources along the $z$ direction at equal intervals, $L$, is considered, the incident field may be written as

$p_{\text {inc }}(\omega, x, y, z)=\frac{2 \pi}{L} \sum_{m=-\infty}^{\infty} \hat{p}_{\text {inc }}\left(\omega, x, y, k_{z}\right) \mathrm{e}^{-\mathrm{i} k_{z m} z}$

with $k_{z m}=(2 \pi / L) m$, that converges and can be approximated by a finite sum of terms.

\section{Boundary element formulation}

The BEM is used to obtain the 3D field generated by a cylindrical inclusion that may be of irregular shape. In the case of an acoustic medium, the 2-1/2D problem can be solved as a discrete summation of 2D BEM problems for different $k_{z}$ wave numbers. It is then possible to synthesise the 3D field using the inverse Fourier transform. As explained above, we can obtain the wave number transform in discrete form, by considering an infinite number of virtual point sources equally spaced along the $z$ axis and sufficiently distant from each other to avoid spatial contamination [19]. In addition, the analyses are done using complex frequencies, shifting down the frequency axis, in the complex plane, in order to minimise the influence of the neighbouring fictitious sources [20].

Given the literature currently available on the BEM, it is unnecessary to describe the formulation needed for the type of scattering problem presented here in detail (see for example, Ref. [21]). It is enough to state that each 2D BEM solution 
requires the evaluation of the integrals

$$
G^{k l}=\int_{C_{l}} \phi G\left(\underline{x}_{k}, \underline{x}_{l}\right) \mathrm{d} C_{l}, \quad H^{k l}=\int_{C_{l}} \phi H\left(\underline{x}_{k}, \underline{x}_{l}, n_{l}\right) \mathrm{d} C_{l},
$$

where $G^{k l}$ and $H^{k l}$ are, respectively, the components of the Green's tensor for pressure and the pressure velocity component at $\underline{x}_{k}$ due to a pressure load at $\underline{x}_{l}, n_{l}$ is the unit outward normal for the $l$ th boundary segment $C_{l}$, and $\phi$ is an interpolation function. In the present case, the required pressure and pressure velocity functions are

$$
\begin{aligned}
& G\left(\underline{x}_{k}, \underline{x}_{l}, n_{l}\right)=-\frac{\mathrm{i}}{4} H_{0}^{(2)}\left(k_{\alpha} r\right), \\
& H\left(\underline{x}_{k}, \underline{x}_{l}, n_{l}\right)=\frac{-\mathrm{i} k_{\alpha}}{4} H_{1}^{(2)}\left(k_{\alpha} r\right) \frac{\partial r}{\partial n_{l}} .
\end{aligned}
$$

In this equation $\rho$ is the mass density, $r$ the source-receiver distance on the plane $x y$, and $\mathrm{i}=\sqrt{-1}$.

If the integral equations are mathematically manipulated, combined and subjected to the continuity conditions at the interface between the two media, and appropriately discretized, a system of equations which can be solved for the nodal pressures and pressure velocities is obtained. The required integrations in Eq. (6) are performed by means of Gauss-Legendre quadrature, using four integration points, when the element to be integrated is not the loaded element. For the loaded element, the existing singular integrands are carried out in closed form [22].

The scattered pressure and velocity pressure fields in the fluid are then defined as a function of the nodal values. As the system of equations obtained is fully populated and generally asymmetrical, most of the global computational cost of the BEM represents the time needed to solve the system of equations. In this paper, boundary elements of different types are used. To evaluate the performance of the different solutions, the results obtained for similar
Table 1

Relations $\lambda / L$ used in the calculations

\begin{tabular}{llrr}
\hline Interpolation function & $R_{1}$ & $R_{2}$ & $R_{3}$ \\
\hline Constant & 6 & 12 & 18 \\
Linear & 3 & 6 & 9 \\
Quadratic & 2 & 4 & 6 \\
\hline
\end{tabular}

computational cost, that is involving equation systems of the same size (i.e. using similar number of nodal points), are compared. Three different relations $\left(R_{1}, R_{2}, R_{3}\right)$ between the wavelength $(\lambda)$ of the pressure waves and the length of the boundary elements $(L)$ were considered, as shown in Table 1. A higher number of elements per wavelength would improve the accuracy of the results. This paper, however, does not aim to define optimal (or practical) computational strategies, but to evaluate the behaviour of different types of boundary elements, at the cost of similar computational effort.

The pressure and pressure velocity variations within a boundary element are defined as a function of the nodal values. The velocity discontinuity existing at the corner between two boundary elements is dealt with using discontinuous boundary elements, which entails moving the nodes that would meet at the corner [23] to the inside. The interpolation functions used to model the inclusions used in the simulation analysis are presented in Fig. 2.

\section{Performance of the BEM solution}

The method used to evaluate the benefit of using higher order elements is the calculation of the pressure field around a cylindrical circular inclusion buried in a homogeneous fluid medium, for which analytical solutions are known. Two inclusions are taken to be submerged in a fluid medium that allows a pressure wave velocity of $\alpha_{1}=1500 \mathrm{~m} / \mathrm{s}$, a fixed cavity and a fluid column cylinder that allows a pressure

Discontinuous BEM elements

Continuous BEM elements

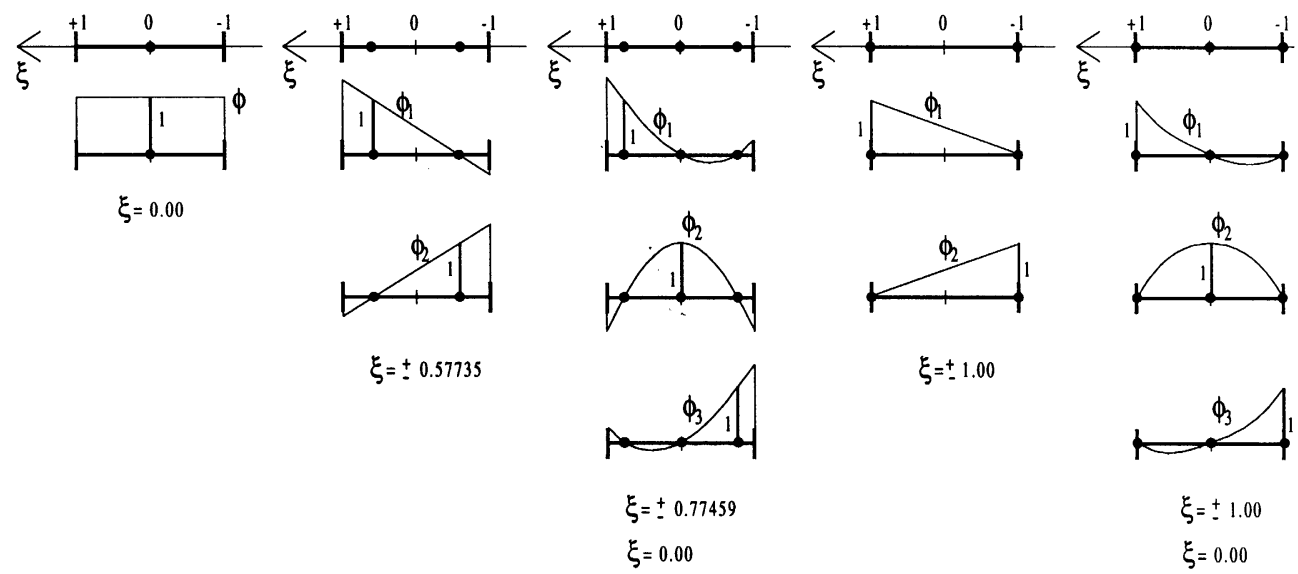

Fig. 2. Interpolation functions and position of the nodal points. 


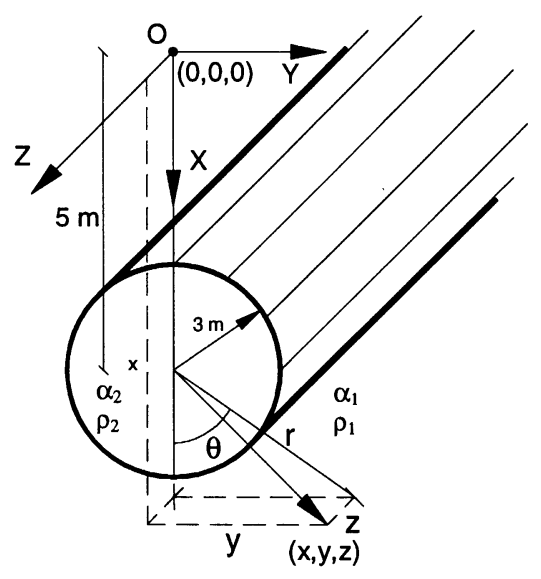

$$
\begin{aligned}
& \text { Outside Fluid } \\
& \begin{array}{l}
\alpha_{1}=1500 \mathrm{~m} / \mathrm{s} \\
\rho_{1}=1000 \mathrm{~kg} / \mathrm{m}^{3} \\
\text { Inside Fluid } \\
\alpha_{2}=2000 \mathrm{~m} / \mathrm{s} \\
\rho_{2}=1000 \mathrm{~kg} / \mathrm{m}^{3}
\end{array}
\end{aligned}
$$

Fig. 3. Circular cylindrical inclusion in an unbounded fluid medium. Medium properties.

wave velocity of $\alpha_{2}=2000 \mathrm{~m} / \mathrm{s}$. Both are illuminated by pressure waves elicited by a point pressure load applied at point $\mathrm{O}$, as in Fig. 3.

\subsection{Analytical solution}

The scattering field produced by a circular cylindrical column of fluid or a fixed cavity, placed in a homogeneous fluid medium, subjected to a point dilatational load can be defined in a circular cylindrical coordinate system $(r, \theta, z)$ and evaluated by using the separation of variables [24].

The frequency and spatial wave number position of the natural modes are found to influence the performance of the BEM. The position of these natural modes, in the systems defined above, can be evaluated, assuming that they include both incoming and outgoing cylindrical waves propagating to and from the cylinder. When the adequate boundary conditions are imposed (null pressure at $r=a$ ) in the presence of this standing field, the response is other than zero if the resulting equation is set to be zero

$J_{n}\left(k_{\alpha 1} r\right)=0$

in which $J_{n}(\cdot)$ are the Bessel functions of the first kind and order $n$, and $k_{\alpha 1}=\sqrt{\omega^{2} / \alpha_{1}^{2}-k_{z}^{2}}$. The solution of the resulting equation gives the required position of the natural modes.

\subsection{Identification of the BEM errors}

Simulation analyses have been performed for a broad range of frequencies and inclusions. However, as it is not possible to present all the results, a restricted number of simulations are used to illustrate the main findings.

Fig. 4 gives the scattering results obtained at one receiver placed at $x=3.5 \mathrm{~m}$ and $y=7.0 \mathrm{~m}$, hereafter referred to as receiver 1 . The results are computed for 1000 frequencies, in the range 1.0-1000 Hz. Both the analytical analysis and the BEM solution were used to calculate the response, discretizing the boundary with constant, linear and quadratic discontinuous elements, as shown in Fig. 2. The positions of the nodal points are the same as those used in GaussLegendre numerical integration (Fig. 2). Pressures were computed for a wide range of wave numbers $k_{z}$, which were then used to obtain the 3D solution by means of (fast) inverse Fourier transform into space. Only the pressure for $k_{z}=0.0 \mathrm{rad} /(\mathrm{m} / \mathrm{s})$ and $k_{z}=1.0 \mathrm{rad} /(\mathrm{m} / \mathrm{s})$ are given here, to illustrate the results. The exact values of the pressure are given in Fig. 4a, while Fig. $4 \mathrm{~b}$ shows the error occurring with the BEM solution when constant, linear and quadratic elements are used. A logarithmic scale is used to show the error, as this enhances the difference in the responses. The relation between the wavelength $(\lambda)$ of the pressure waves and the length of the boundary elements $(L)$ was considered to be $R_{2}$, as listed in Table 1 . In no case is the number of nodal points less than 24. The positions of the lower natural modes, given by solving Eq. (7), are also displayed.

Analysis of the results shows that the BEM is considerably less accurate at frequencies in the vicinity of the natural modes, where large peaks of error are found. This is a wellknown behaviour that results from the lack of solution uniqueness of the surface Helmholtz integral formulation $[25,26]$, for which different integral formulations have been proposed to improve the results.

The constant elements are noticeably poorer in these localised frequency domains. All the responses outside these domains improve with the increase of the number of nodal points, as expected, because a BEM model with a large number of nodes can model variations in pressure and/or pressure velocity much more realistically. It was also observed that the error peaks shift their position towards the values of the frequencies satisfying Eq. (7) as the relation between the wavelength $(\lambda)$ of the pressure waves and the length of the boundary elements $(L)$ increased (not illustrated here). The fact that increasing the number of 

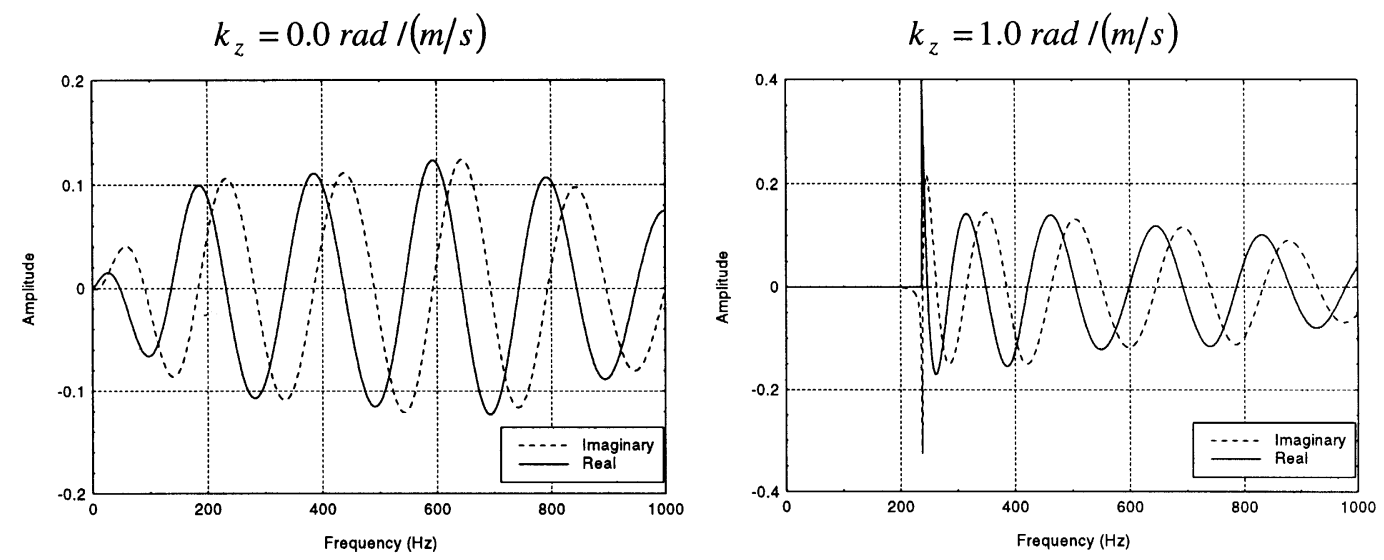

a)
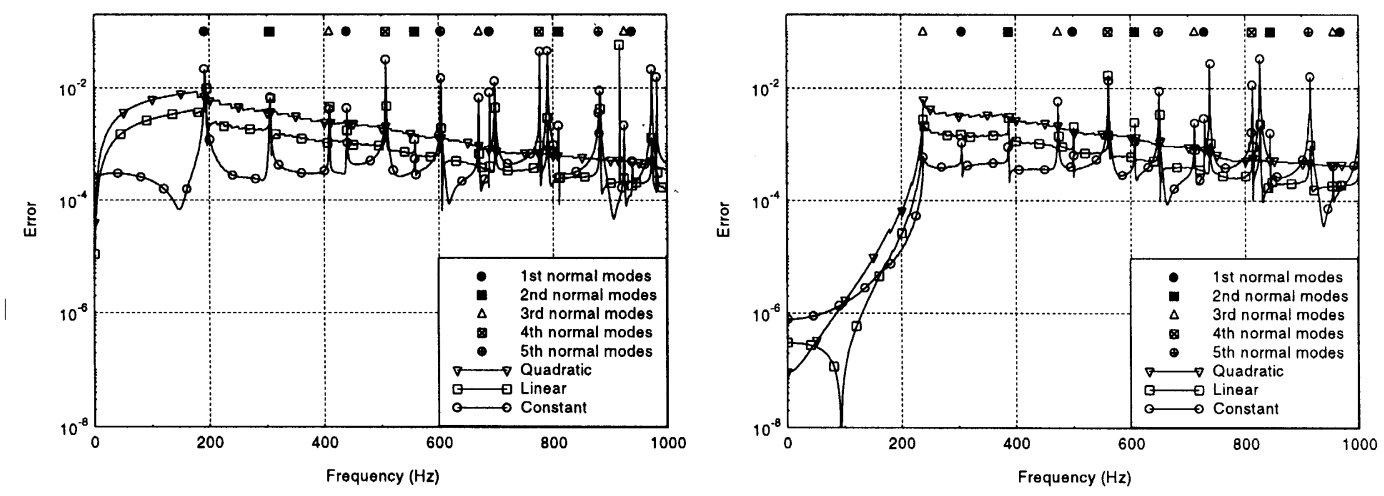

b)

Fig. 4. Scattering response at receiver $1\left(k_{z}=0.0 \mathrm{rad} /(\mathrm{m} / \mathrm{s})\right.$ and $\left.k_{z}=1.0 \mathrm{rad} /(\mathrm{m} / \mathrm{s})\right)$ : (a) analytical solution; (b) BEM error.

elements allows the BEM model to approach the definition of the circular cylinder better, with respect to its dynamic behaviour, explains this.

Furthermore, for higher relations of $\lambda / L$ (such as $R_{2}$ ), the BEM errors obtained using linear and quadratic elements are seen to have a global tendency to decrease as the frequency increases. In other words, as more boundary elements are required for the higher excitation frequencies than for the lower ones to satisfy a specific relation of $\lambda / L$, one can expect a higher degree of accuracy for those frequencies because the number of elements is itself an important factor.

To get a better picture of the effect of the influence of the natural modes on the BEM solution, the scattering response was subsequently calculated over a fine grid. In the example below, the inclusion is illuminated by a pulsating source vibrating at a single frequency at $k_{z}=1.0 \mathrm{rad} /(\mathrm{m} / \mathrm{s})$, which is either $473.3 \mathrm{~Hz}$ or $420 \mathrm{~Hz}$. The former frequency corresponds to one of the natural modes of the real system (Eq. (7)) and the latter to a frequency outside the vicinity of any eigenfrequency. The inclusion is modelled with discontinuous elements, using constant, linear and quadratic interpolation functions.

The number of elements was chosen so that results evaluated at similar computational cost (i.e. the same number of nodal points) could be compared. In these calculations, three different ratios between the wavelength of the dilatational waves and the length of the boundary elements were considered, as listed in Table 1.

Fig. 5 shows the analytical response and the modulus of the error occurring with the BEM solution for a frequency of $473.3 \mathrm{~Hz}$, when different relations $\lambda / L$ are used $\left(R_{1}, R_{2}\right.$, and $R_{3}$ ). They show that the use of constant elements to model the inclusion gives poor agreement between the BEM and the analytical solution (see Fig. 5b). Notice that the BEM error appears to be significant when compared with the analytical solution. It can further be observed that refining the boundary elements (i.e. changing their number) does not lead to significant improvement in the BEM solution. Indeed, as Fig. 5b shows, the use of 107 constant elements to model the inclusion results in an error similar to that given when the inclusion is modelled with 71 elements. The quadratic elements seem to perform poorly in the vicinity of the inclusion for a low number of nodal points (33) (see Fig. 5d). As the number of elements is increased, there is a rapid improvement in the quadratic elements, and a response calculated with 105 nodal points yields a performance approaching that of the linear elements (see Fig. 5c and d). 


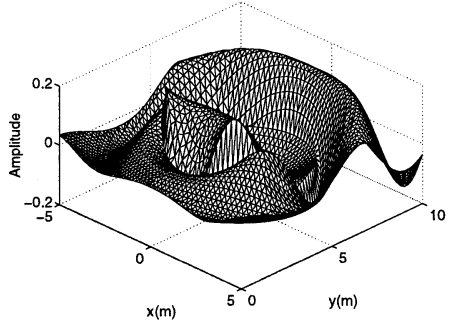

Real part

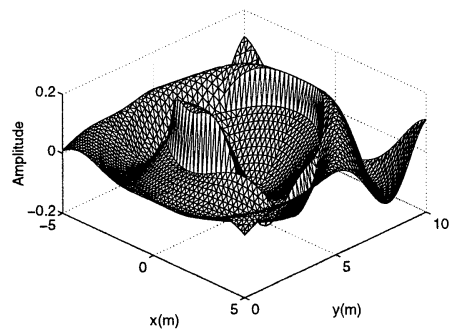

Imaginary part a)

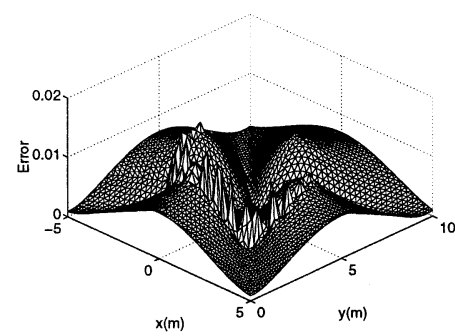

35 elements

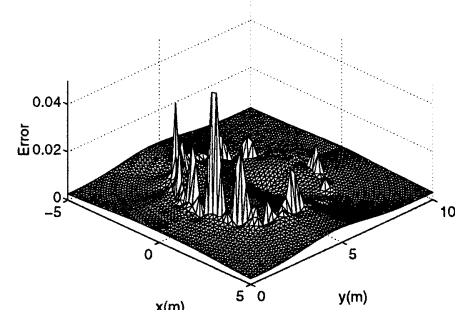

17 elements

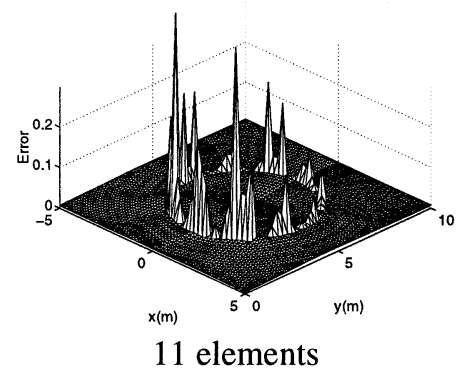

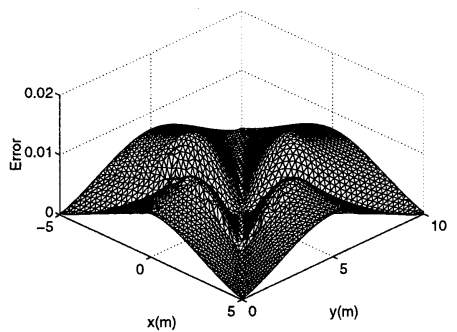

71 elements

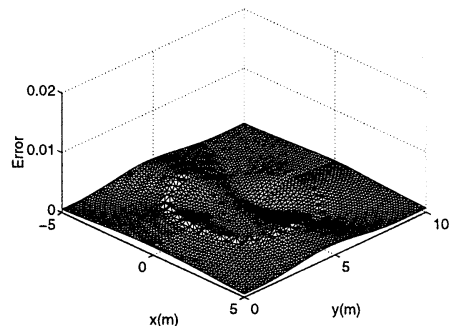

35 elements

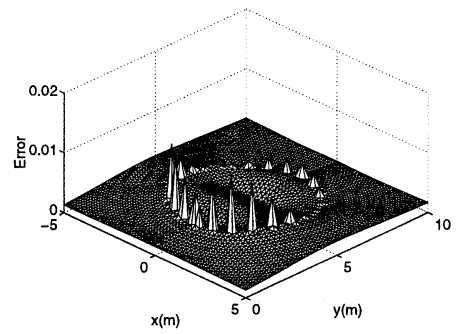

23 elements

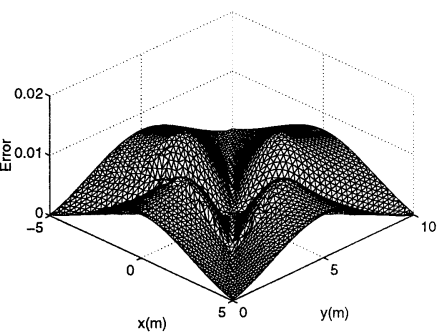

107 elements

b)

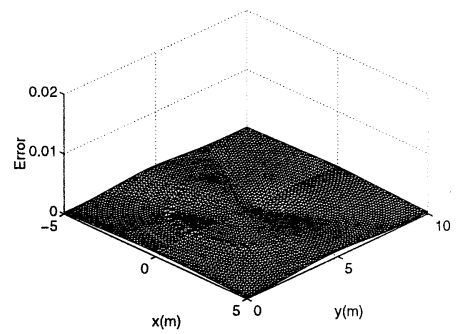

53 elements

c)

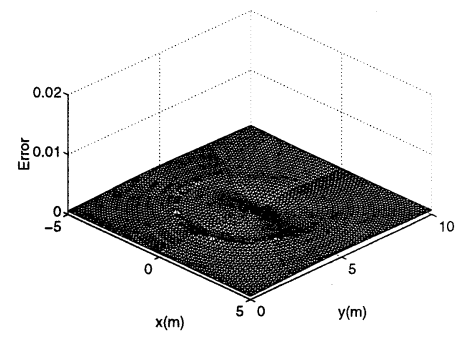

35 elements

Fig. 5. Scattered pressure in the presence of a cylindrical column of fluid $\left(k_{z}=1.0 \mathrm{rad} /(\mathrm{m} / \mathrm{s})\right)$. Frequency of excitation $-473.3 \mathrm{~Hz}$ : (a) analytical solution; (b) modulus of the BEM error - constant elements; (c) modulus of the BEM error — discontinuous linear elements; (d) modulus of the BEM error discontinuous quadratic elements.

Fig. 6a shows the analytical response and the BEM error when the vibrating source is excited with a frequency of $420 \mathrm{~Hz}$, while Fig. $6 \mathrm{~b}-\mathrm{d}$ shows the BEM error when the number of the boundary elements used to model the inclusion satisfies the relation $\lambda / L$ set to $R_{3}$ (see Table 1). Again, each plot is the result of computations performed with a similar number of nodal points, which is assumed to require similar computational cost. These results, which are very different from the previous ones (i.e. those evaluated for a frequency of $473.3 \mathrm{~Hz}$ ), contain a number of interesting features. We now have a much-improved performance from the constant elements, giving better results than the linear and quadratic interpolating functions.

The scattered response was subsequently evaluated around a fixed cavity over the same grid, in the presence of the same harmonic vibrating sources $(473.3$ or $420 \mathrm{~Hz}$ ). At the nodal points the unknowns are limited to the pressures, and so the use of continuous elements is allowed. Fig. 7a presents the analytical solution for the pressure 


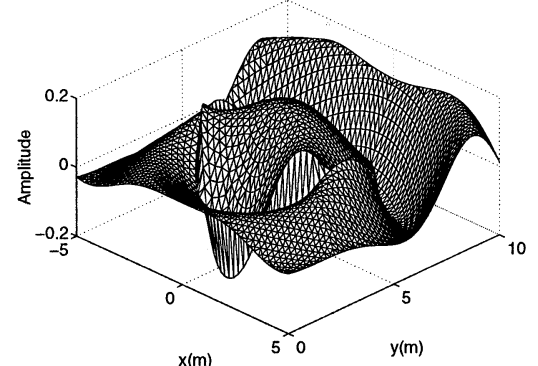

Real part

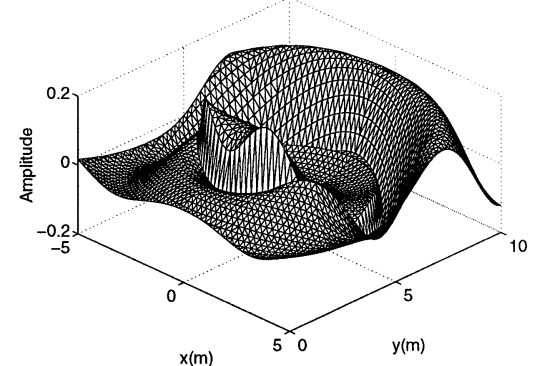

Imaginary part a)

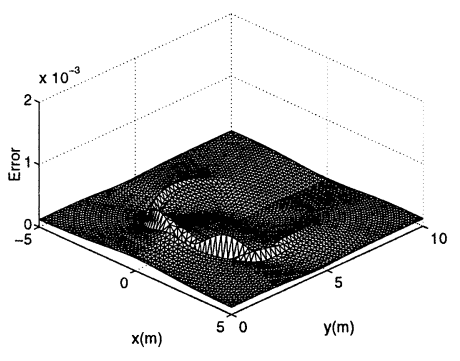

95 elements

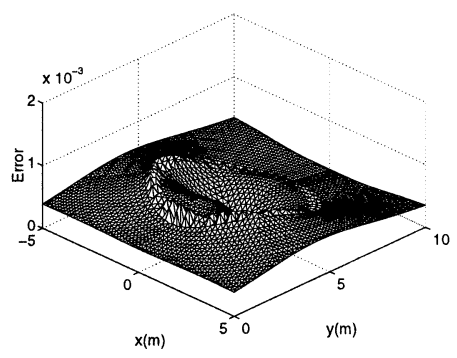

47 elements

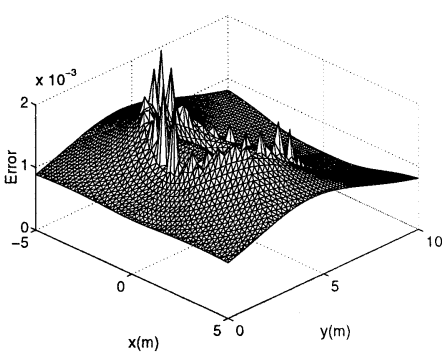

31 elements

d)

Fig. 6. Scattered pressure in the presence of a cylindrical column of fluid $\left(k_{z}=1.0 \mathrm{rad} /(\mathrm{m} / \mathrm{s})\right)$. Frequency of excitation $-420.0 \mathrm{~Hz}$ : (a) analytical solution; (b) modulus of the BEM error - constant elements; (c) modulus of the BEM error - discontinuous linear elements; (d) modulus of the BEM error discontinuous quadratic elements.

field when the source is excited with a frequency of $473.3 \mathrm{~Hz}$ at $k_{z}=1.0 \mathrm{rad} /(\mathrm{m} / \mathrm{s})$, while Fig. $7 \mathrm{~b}-\mathrm{f}$ shows the modulus of the BEM error when the fixed cavity is modelled with a number of elements that verifies the relation $\lambda / L$ set to $R_{3}$ (see Table 1). Analysis of the results reveals that the continuous linear and constant elements do not perform well when the source is excited with a frequency of $473.3 \mathrm{~Hz}$. Again, for a low number of nodal points the continuous and discontinuous quadratic elements perform poorly (not displayed), especially in the vicinity of the

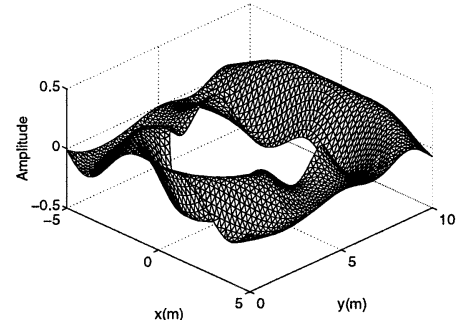

Analytical

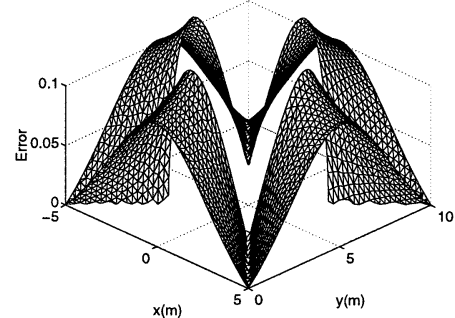

107 elements

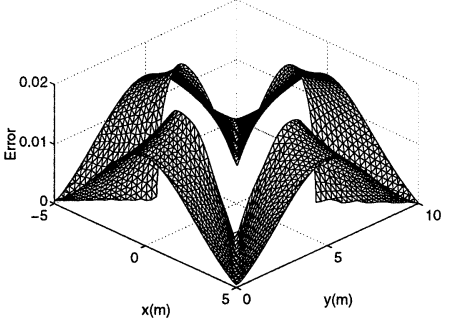

107 elements

b)

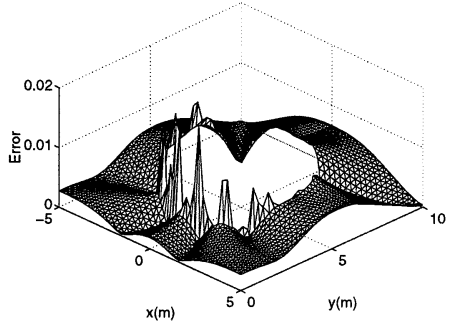

35 elements

e)

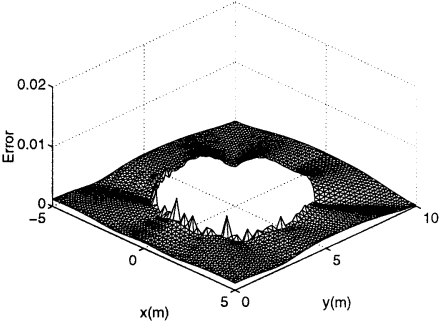

53 elements

c)

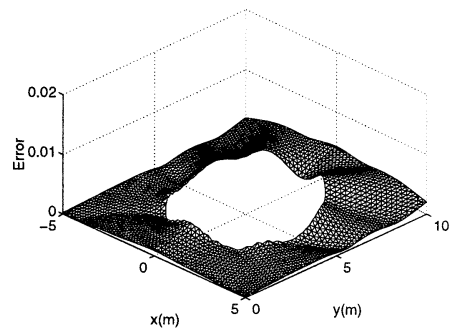

53 elements

f)

Fig. 7. Scattered pressure in the presence of a fixed cylindrical cavity $\left(k_{z}=1.0 \mathrm{rad} /(\mathrm{m} / \mathrm{s})\right)$. Frequency of excitation $-473.3 \mathrm{~Hz}$ : (a) analytical solution; (b) modulus of the BEM error — constant elements; (c) modulus of the BEM error — discontinuous linear elements; (d) modulus of the BEM error continuous linear elements; (e) modulus of the BEM error — discontinuous quadratic elements; (f) modulus of the BEM error — continuous quadratic elements. 

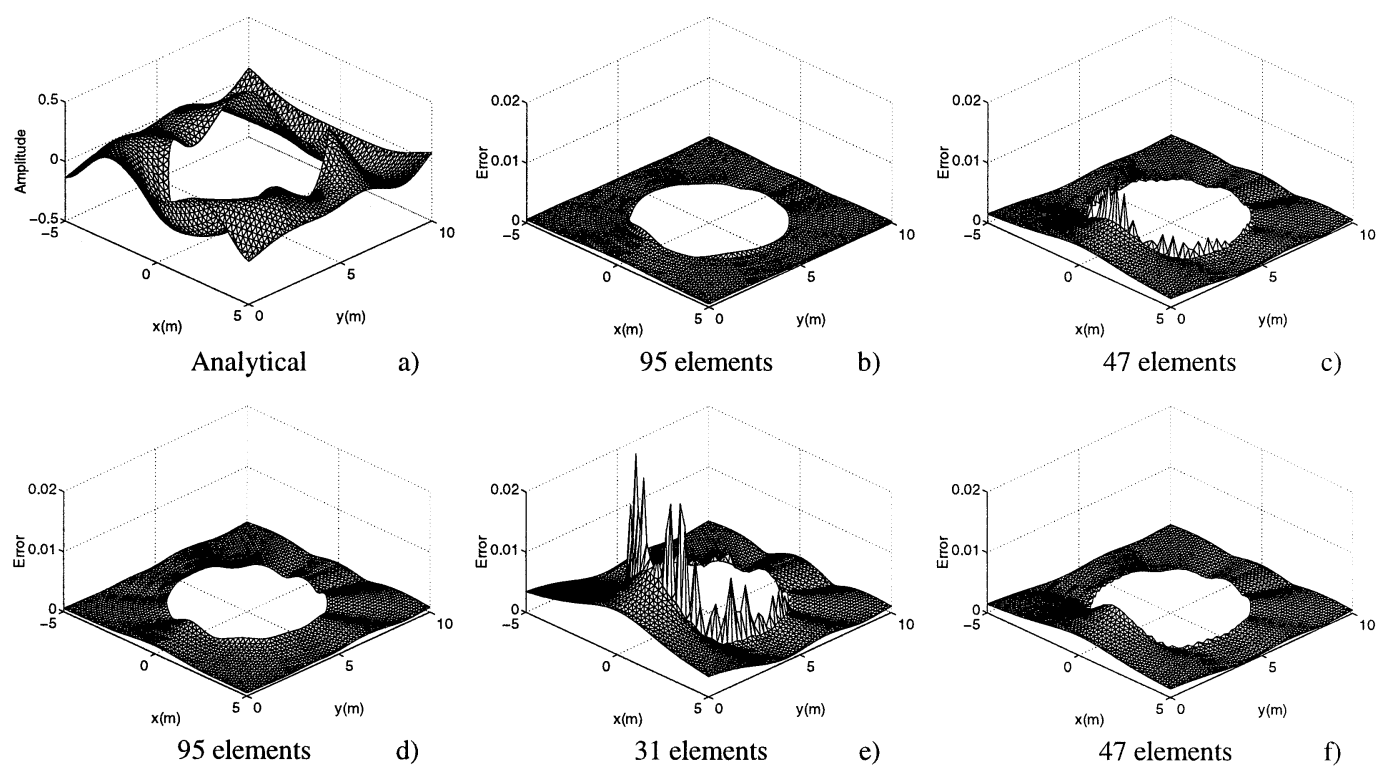

Fig. 8. Scattered pressure in the presence of a fixed cylindrical cavity $\left(k_{z}=1.0 \mathrm{rad} /(\mathrm{m} / \mathrm{s})\right)$. Frequency of excitation $-420.0 \mathrm{~Hz}$ : (a) analytical solution; (b) modulus of the BEM error - constant elements; (c) modulus of the BEM error - discontinuous linear elements; (d) modulus of the BEM error continuous linear elements; (e) modulus of the BEM error - discontinuous quadratic elements; (f) modulus of the BEM error - continuous quadratic elements.

inclusion, improving rapidly as the number of the elements increases. When the number of elements verifies the relation $\lambda / L$ set to $R_{3}$, the continuous quadratic elements are found to be better, even at points in the vicinity of the fixed cavity surface. Fig. 8 presents the modulus of the error for a frequency of $420.0 \mathrm{~Hz}$. As was found for the column of fluid inclusion, the results differ markedly from those evaluated for a frequency of $473.3 \mathrm{~Hz}$. The quadratic elements show greater divergence from the analytical solution, while the constant and continuous linear elements now give good results.

The examples described focus on how the BEM behaves when a source with a specific frequency and wave number is excited. Indeed, as shown previously, the 3D solution in frequency demands that a broad range of wave numbers be calculated (see Eq. (4)), while the time solution also requires the response for a set of frequencies. To illustrate the performance of the BEM in the resolution of this problem, simulation analyses are next performed to calculate the full 3D response at receiver 1. Computations are performed in the frequency range $(10,750 \mathrm{~Hz})$, with a frequency increment of $10 \mathrm{~Hz}$. The spatial period considered in the analysis is $L=2 T \alpha=300 \mathrm{~m}$. A number of boundary elements, changing with the frequency of excitation of the harmonic load satisfying the ratios $R_{1}$ and $R_{3}$, are used to model the cavity (see Table 1 ). The minimum number of nodal points is never less than 24. Fig. 9a shows the amplitude of the analytical pressure in the frequency vs wave number domain at receiver 1 . Notice that values of $k_{z}$ in excess of $\omega / \alpha$ correspond to heterogeneous, evanescent waves, which decay rapidly in space. When the relation $\lambda / L$ assumes the values $R_{1}$ and $R_{3}$, respectively, the BEM errors, illustrated in Fig. $9 \mathrm{~b}-\mathrm{f}$ have features similar to those observed in the previous cases. When the number of boundary elements is increased, the position of the peak errors in these plots agrees with the solutions given by Eq. (7). The discontinuous quadratic elements perform poorly when the number of elements is defined by the ratio $R_{1}$ (see Fig. 9e). At lower frequencies and wave numbers, the number of elements used to define the inclusion is an important factor. Indeed, at lower frequencies the constant elements uses 24 elements, while the discontinuous linear and discontinuous quadratic elements use 12 and 8 boundary elements, respectively, thus there is a better definition of the inclusion when it is modelled by constant elements. As the frequency increases, a higher number of elements is required to satisfy a specific $\lambda / L$ relation, leading to an improvement in the linear and quadratic elements. Again, constant boundary elements are outperformed by the discontinuous linear elements in the vicinity of the natural modes. Fig. $9 \mathrm{~b}$ and d exhibits a peak valley

Fig. 9. Scattered pressure in the presence of a fixed cylindrical cavity at receiver 1 in the frequency versus wave number domain; (a) analytical solution; (b) modulus of the BEM error $\left(R_{1}\right.$ and $\left.R_{3}\right)$ - constant elements; (c) modulus of the BEM error $\left(R_{1}\right.$ and $\left.R_{3}\right)$ - discontinuous linear elements; (d) modulus of the BEM error $\left(R_{1}\right.$ and $\left.R_{3}\right)$ - continuous linear elements; (e) modulus of the BEM error $\left(R_{1}\right.$ and $\left.R_{3}\right)$ - discontinuous quadratic elements; (f) modulus of the BEM error $\left(R_{1}\right.$ and $\left.R_{3}\right)$ - continuous quadratic elements. 


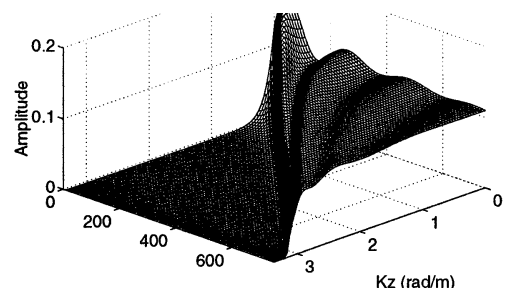

Frequency $(\mathrm{Hz})$

$\lambda / L=R_{1}$

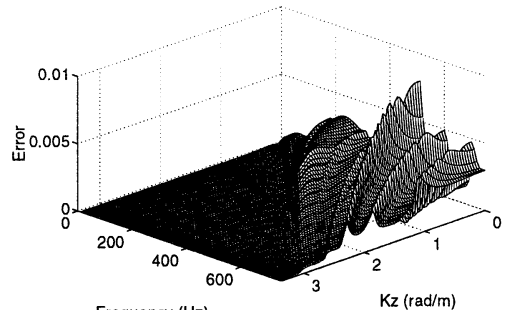

Frequency ( $\mathrm{Hz})$
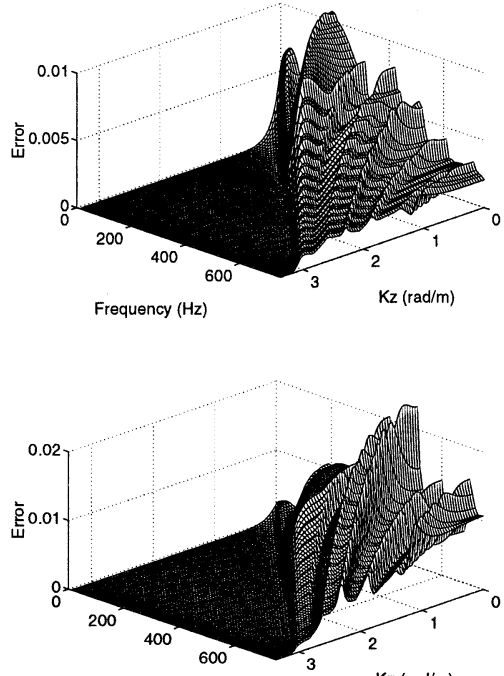

Frequency $(\mathrm{Hz})$

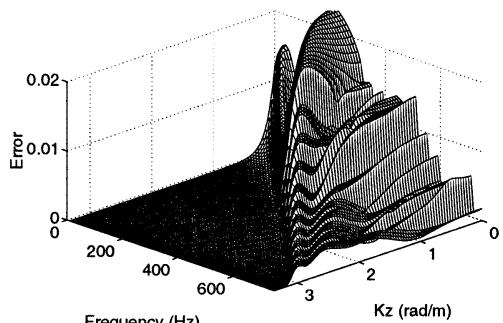

Frequency $(\mathrm{Hz})$

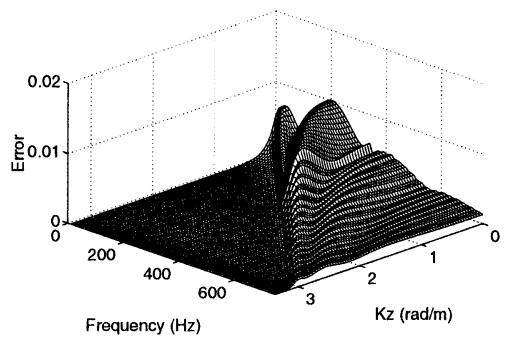

$\lambda / L=R_{3}$

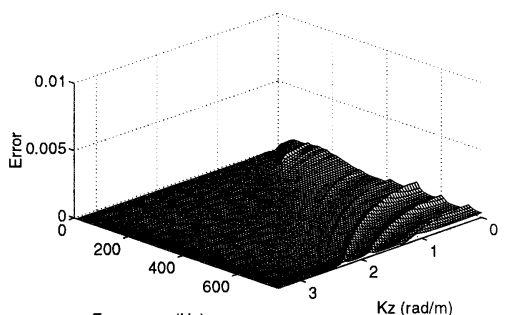

b)

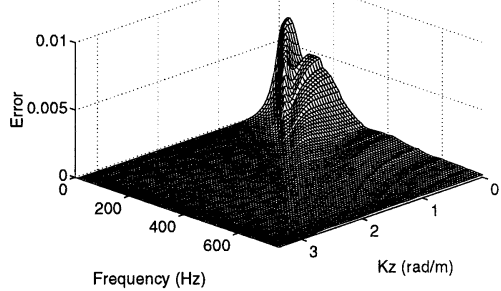

c)

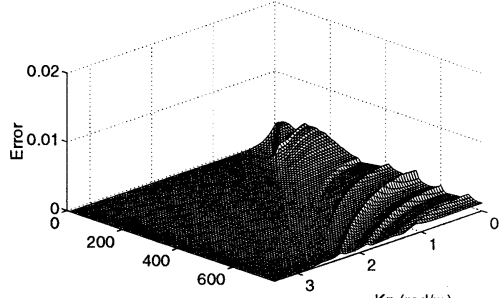

Frequency $(\mathrm{Hz})$

$\mathrm{Kz}(\mathrm{rad} / \mathrm{m})$

d)

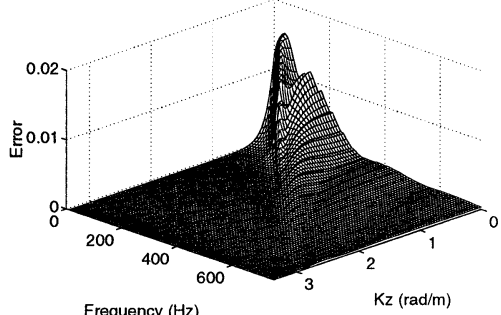

e)

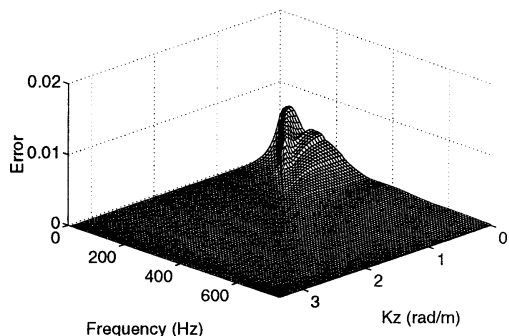


structure error that follows the position of the natural modes.

\section{Importance of the nodal points position}

In the above examples the nodal points placed along the discontinuous elements were chosen to coincide with those in a Gauss-Legendre numerical integration. The results given by simulations performed using different positions of the nodal points seem to indicate that this is a good choice. To demonstrate this assertion, consider the same wave propagation problem described earlier. Once again, the scattered field is computed for a cylindrical circular fixed cavity over a fine grid placed around the inclusion. Fig. 10 shows the BEM error for the pressure for a frequency of $473.3 \mathrm{~Hz}$, and a relation of $\lambda / L$ set to $R_{3}$ (see Table 1), for different locations of nodal points around those given by the Gauss-Legendre numerical integration and also when the inclusion is modelled with linear and quadratic elements. The interior nodal point of the quadratic elements is placed at the centre of the boundary segment in all the analyses. The position of the nodal points has a considerable influence on the accuracy obtained with the linear elements. It can be seen that moving the nodal points to the positions used in the Gauss-Legendre integration improves the solution. Moving the nodal points around the vicinity of the Gauss-Legendre numerical integration points does not seem to significantly affect the results from the quadratic elements. Nevertheless, the BEM solution clearly loses accuracy as the nodal points are moved closer to the extremity of the boundary element.

a)

b)
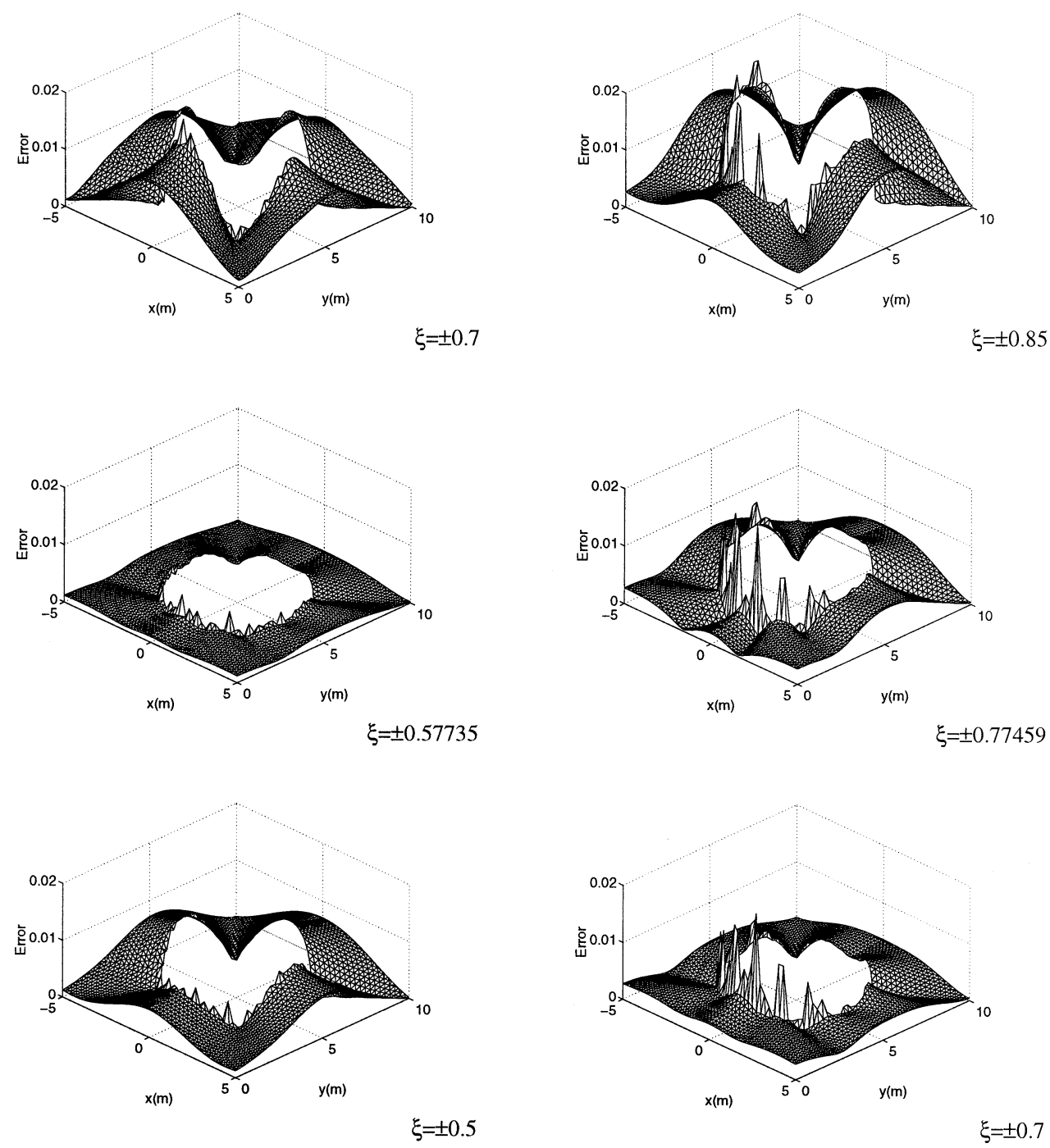

Fig. 10. Scattered pressure in the presence of a fixed cylindrical cavity $\left(k_{z}=1.0 \mathrm{rad} /(\mathrm{m} / \mathrm{s})\right)$ when the nodal points are placed at different positions inside each element. Frequency of excitation $-473.3 \mathrm{~Hz}$ : (a) modulus of the BEM error $\left(R_{3}\right)$ - discontinuous linear elements; (b) modulus of the BEM error $\left(R_{3}\right)-$ discontinuous quadratic elements. 
Near singular integrals would occur when integrating over neighbouring elements, if the nodal points were placed very close to the extremity of the elements. Notice, that the correctness of the required integrals has been checked by testing the performance of the solution for a higher number of integration points, confirming that the appropriate number of Gaussian integrating points was used. Analytical solutions were used when the element to be integrated was the loaded element.

Simulation analysis performed when the source vibrates at $420.0 \mathrm{~Hz}$ (not illustrated here) show similar behaviour. The BEM error was subsequently tested for cylindrical circular cavities of differing sizes, for a broad range of frequencies and spatial wave numbers, and these showed similar conduct.

\section{Conclusions}

This paper has assessed the benefits of using constant, linear and quadratic interpolation functions to model 3D fluid environments. Circular cylindrical models submerged in a fluid medium, namely a fixed cavity and a column of fluid, were used to conduct simulation analyses. The number of boundary elements used changed according to the different relations between the wavelength $(\lambda)$ of the incident waves and the length of the boundary elements $(L)$.

Comparison of the BEM results with those obtained by analytical solution showed that it is important to use linear discontinuous elements rather than constant and linear continuous elements to calculate the pressure field. The discontinuous linear boundary elements have been shown to outperform the constant elements, especially at frequencies in the vicinity of natural modes. The number of boundary elements used to model an inclusion appears to be an important factor, as well as the relation between the wavelength and the boundary elements size $(\lambda / L)$. The quadratic elements use a lower number of elements to verify a specific number of nodal points than the other types of elements do. At lower frequencies this number of elements is too low when the relation $\lambda / L$ is small, leading to a poor performance. As the frequency increases, the performance of the quadratic elements improves rapidly, because more boundary elements are required, and it even surpasses that yielded by other types of elements.

The position of the nodal points inside the discontinuous linear elements was also observed to have a considerable influence on the accuracy of the solution at frequencies in the vicinity of the natural modes of vibration. It was found that locating the nodal points to coincide with the GaussLegendre numerical integration points yielded good results.

\section{References}

[1] Trifunac MD. Surface motion of a semi-cylindrical alluvial valley for incident plane SH waves. Bull Seismol Soc Am 1971;61:1755-70.
[2] Lee VW. Three-dimensional diffraction of elastic waves by a spherical cavity in an elastic half-space. 1: closed-form solutions. Soil Dyn Earthq Engng 1988;7:149-61.

[3] Lee VW, Karl JA. Diffraction of SV waves by underground circular, cylindrical cavities. Soil Dyn Earthq Engng 1992;11:445-56.

[4] Sanchez-Sesma FJ. Diffraction of elastic waves by three dimensional surface irregularities. Bull Seismol Soc Am 1983;73:1621-36.

[5] Kausel E. Forced vibrations of circular foundations in layered media, MIT Research Report 70-3, Department of Civil Engineering, Massachusetts Institute of Technology, Cambridge, MA, 1974.

[6] Ohtsuki A, Harumi K. Effect of topography and subsurface inhomogeneities on seismic SV waves. Earthq Engng Struct Dyn 1983;11:441-62.

[7] Dawson TW, Fawcett JA. A boundary integral equation method for acoustic scattering in a waveguide with nonplanar surfaces. J Acoust Soc Am 1990;87:1110-25.

[8] Fawcett JA. The computational of the scattered pressure field from a cylinder embedded between two half-spaces with different densities. J Acoust Soc Am 1996;99:2435-8.

[9] Fawcett JA. Acoustic scattering from cylindrical objects embedded between two half-spaces. J Acoust Soc Am 1996;100:3053-60.

[10] Lacerda LA, Wrobel LC, Power H, Mansur WJ. A novel boundary integral formulation for three-dimensional analysis of thin acoustic barriers over an impedance plane. J Acoust Soc Am 1998;104:671-8.

[11] Shah AH, Wong KC, Datta SK. Diffraction of plane SH waves in a half-space. Earthq Engng Struct Dyn 1982;10:519-28.

[12] Lim R. Acoustic scattering by a partially buried three-dimensional elastic obstacle. J Acoust Soc Am 1998;104:769-82.

[13] Beskos DE. Wave propagation through ground. In: Manolis GD, Davies TG, editors. Boundary element techniques in geomechanics. Southampton/London: Computational Mech. Publ./Elsevier, 1993. p. 359-406.

[14] Sanchez-Sesma FJ, Campillo M. Topographic effects for incident $P$, SV and Rayleigh waves. Tectonophysics 1993;218:113-25.

[15] Wong HL, Jennings PC. Effect of canyon topographies on strong ground motion. Bull Seismol Soc Am 1975;65:1239-57.

[16] Tadeu AJB, Kausel E, Vrettos C. Scattering of waves by subterranean structures via the boundary element method. Soil Dyn Engng, 1996;15:387-97.

[17] Tadeu AJB, Santos PFA. Scattering of waves by buried cavities via boundary element method. EUROBEM, Wessex Institute of Technology, Southampton, UK, 1998. p. 189-200.

[18] Santos P, Tadeu A. Scattering of waves by buried inclusions via boundary element method. Proceedings of the XXV IAHS World Housing Congress, International Association for Housing Science, Lisbon, Portugal, 1998. p. 655-64.

[19] Bouchon M, Aki K. Discrete wave-number representation of seismicsource wave field. Bull Seismol Soc Am 1977;67:259-77.

[20] Phinney RA. Theoretical calculation of the spectrum of first arrivals in layered elastic mediums. J Geophys Res 1965;70:5107-23.

[21] Manolis GD, Beskos DE. Boundary element methods in elastodynamics. London: Unwin Hyman, 1988 (sold to Chapman \& Hall).

[22] Tadeu A, Santos P, Kausel E. Closed-form integration of singular terms for constant, linear and quadratic boundary elements. Part I: SH wave propagation. Engng An Bound Elmts 1999;23(8):67181.

[23] Brebbia CA, Telles JC, Wrobel LC. Boundary elements techniques, Theory and applications in engineering. Berlin: Springer, 1984.

[24] Pao YH, Mow CC. Diffraction of elastic waves and dynamic stress concentrations. New York: Crane and Russak, 1973.

[25] Burton AJ, Miller GF. The application of integral equation methods to the numerical solution of some exterior boundary-value problems. Proc R Soc London 1971;323:201-10.

[26] Schneck HA. Improved integral formulation for acoustic radiation problems. J Acoust Soc Am 1968;44:41-58. 